\title{
Modeling of Fission Neutrons as a Signature for Detection of Highly Enriched Uranium
}

James K. Wolford Jr., Michael I. Frank, Marie-Anne Descalle

This article was submitted to SPIE 2004 Defense and Security Symposium Orlando, Florida, April 12-16, 2004

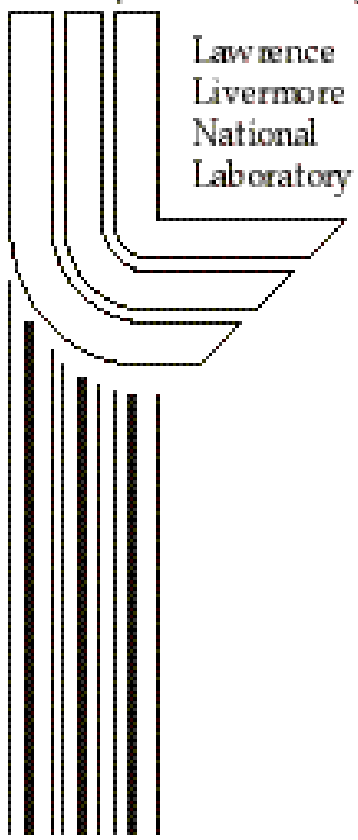

March, 2004 


\section{DISCLAIMER}

This document was prepared as an account of work sponsored by an agency of the United States Government. Neither the United States Government nor the University of California nor any of their employees, makes any warranty, express or implied, or assumes any legal liability or responsibility for the accuracy, completeness, or usefulness of any information, apparatus, product, or process disclosed, or represents that its use would not infringe privately owned rights. Reference herein to any specific commercial product, process, or service by trade name, trademark, manufacturer, or otherwise, does not necessarily constitute or imply its endorsement, recommendation, or favoring by the United States Government or the University of California. The views and opinions of authors expressed herein do not necessarily state or reflect those of the United States Government or the University of California, and shall not be used for advertising or product endorsement purposes.

This is a preprint of a paper intended for publication in a journal or proceedings. Since changes may be made before publication, this preprint is made available with the understanding that it will not be cited or reproduced without the permission of the author.

This report has been reproduced directly from the best available copy.

Available to DOE and DOE contractors from the Office of Scientific and Technical Information

P.O. Box 62, Oak Ridge, TN 37831

Prices available from (423) 576-8401 http://apollo.osti.gov/bridge/

Available to the public from the National Technical Information Service

U.S. Department of Commerce 5285 Port Royal Rd., Springfield, VA 22161 http://www.ntis.gov/

OR

Lawrence Livermore National Laboratory Technical Information Department's Digital Library http://www.llnl.gov/tid/Library.html 


\title{
Modeling of Fission Neutrons as a Signature for Detection of Highly Enriched Uranium
}

\author{
James K. Wolford, Jr. ${ }^{\dagger}$, Michael I. Frank ${ }^{\ddagger}$, and Marie-Anne Descalle* \\ Lawrence Livermore National Laboratory, P.O. Box 808, Livermore, CA 94551
}

\begin{abstract}
We present the results of modeling intended to evaluate the feasibility of using neutrons from induced fission in highly enriched uranium (HEU) as a means of detecting clandestine HEU, even when it is embedded in absorbing surroundings, such as commercial cargo. We characterized radiation from induced fission in HEU, which consisted of delayed neutrons at all energies and prompt neutrons at energies above a threshold. We found that for the candidate detector and for the conditions we considered, a distinctive HEU signature should be detectable, given sufficient detector size, and should be robust over a range of cargo content. In the modeled scenario, an intense neutron source was used to induce fissions in a spherical shell of HEU. To absorb, scatter, and moderate the neutrons, we place one layer of simulated cargo between the source and target and an identical layer between the target and detector. The resulting neutrons and gamma rays are resolved in both time and energy to reveal the portion arising from fission. We predicted the dominant reaction rates within calcium fluoride and liquid organic scintillators. Finally, we assessed the relative effectiveness of two common neutron source energies.
\end{abstract}

Keywords: fissile material, radiation detection, threat reduction, neutron interrogation

\section{BACKGROUND}

Many projects at U.S. Government laboratories are addressing the problem of detecting highly enriched uranium (HEU) inside containers of commercial cargo. Lawrence Livermore National Laboratory (LLNL) has created an experimental test bed for researching potential detection signatures using (among other techniques) active interrogation with neutrons. Ongoing experiments are testing the effectiveness of various sources, detectors, and data analysis methods. ${ }^{1}$ However, the wide range of possible combinations of cargo and clandestine objects makes comprehensive experimentation impossible. Computational modeling offers a way to extend and give context to this experimental foundation. Accordingly, we created a computational capability to mirror the experimental one. Beyond merely predicting and reproducing experimental results, we identified five challenges that make detecting HEU within cargo containers an interesting problem:

1. Lack of knowledge ahead of time of the object to be found: unknown identity, size, and location of the HEU to be detected and unknown quantity and composition of absorbing/scattering material between source and HEU object and between HEU object and detector. Other special purpose detection systems (such as in arms control and treaty verification) have been allowed to make assumptions about both the assayed object and the intervening material - a luxury one does not enjoy in a screening search for HEU.

2. As a consequence of 1 , potential difficulty in getting enough neutron fluence on target to induce a distinctive signature, and difficulty in capturing that signature in a detector. Surveys of cargo makeup show that a large fraction (by weight) of cargo entering the U.S. consists of foodstuffs and tree products, i.e., materials rich in hydrogen. Such material strongly scatters and absorbs neutrons, and many mean free paths of it may exist in the measurement scenarios under consideration.

3. Inadequate representation in cross section data of the relevant physics underlying the signature. Historically, radiation transport codes needed to model two extremes well: thermal neutrons and D-T fusion $(14 \mathrm{MeV})$ neutrons. Evaluated cross section tables show less concern for the intermediate energy range.

\footnotetext{
†e-mail: wolford@1lnl.gov; telephone: (925) 422-7236; fax: (925) 423-2759

te-mail: mfrank@1lnl.gov; telephone: (925) 424-5853; fax: (925) 423-2759

*e-mail: descalle1@1lnl.gov; telephone: (925) 423-9660; fax: (925) 424-2778
} 
4. Competing signatures from trace radioactivity in the cargo being screened. Typical sources include ${ }^{\text {nat }} \mathrm{U}$, ${ }^{\text {nat }} \mathrm{Th}$, and

${ }^{40} \mathrm{~K}$. In the active interrogation system we are modeling, one must also contend with induced activity after a source pulse.

5. The limited ability of real detectors to distinguish the sought after particle type, particle energy, and time window for the signature of interest.

This study addresses concerns $1,2,3$, and 5 . Since we focused on neutron signatures, we have not tried to represent the trace radiation from the cargo material, which should consist entirely of gamma rays.

Thermal neutron-induced prompt fission in HEU yields, on average, 2.4 neutrons (with most probable energy near 1 $\mathrm{MeV}$ ) and 8 gamma rays per fission. In addition, roughly $0.65 \%$ of all neutrons from induced fissions are emitted at times ranging from fractions of a second to minutes after the fissions take place. When they appear, delayed fission neutrons exhibit an energy distribution that peaks at approximately $0.6 \mathrm{MeV}$, accompanied by gamma-ray emission. Special purpose detection systems ${ }^{\dagger}$ have evolved that use one or both forms of radiation to infer the presence and identity of fissile material being interrogated. In this study, we will analyze the fluence of neutrons into the detector for time, energy, and spatial characteristics and identify two fission signatures involving only detected neutrons. A broader treatment would also consider signatures from the gammas or from a combination of neutrons and gammas.

The brevity of this study necessarily limited its scope. Therefore we chose not to address matters of absorbed dose by humans in the vicinity of the source or the irradiated cargo. Also, we made no attempt to optimize the interrogation of the target or the subsequent signature detection.

\section{DESCRIPTION OF THE TRANSPORT MODEL}

The modeling software used in this study was the LLNL COG program. ${ }^{2}$ COG is a 3-dimensional simulation code for coupled transport of neutrons, protons, gamma rays, and electrons using the Monte $\mathrm{Carlo}^{3}$ method. It includes detailed nuclear reaction physics, including a data-driven treatment of delayed radiation due to the $\beta$-decay of fission products. That is, the algorithm is able to create both neutrons and gammas from the decay of fission products; however, it depends on the completeness of the decay description in the cross section tables for the many nuclides that fission produces. The user can specify detector response functions to modify the radiation tallies so that they simulate measurements from actual instruments with finite resolutions and energy-dependent efficiencies. The ENDF/B-VI library is the most complete compilation of this data to date; however, it accounts only for the delayed neutrons and not for the delayed gammas produced directly by nuclear de-excitation of fission products. Thus the gamma spectra predicted at late time are incomplete, in that they consist only of contributions from capture of, and fission from, the delayed neutrons that are tabulated. COG predicts radiation flux in user-prescribed "detectors," which can be volumes, surfaces, or geometric points. COG also has an option that allows the user to log the particles as they traverse surfaces in the geometry, recording the particle's position, momentum, energy, statistical weight, and age since the birth of the particle (or the birth of the source particle that reacted to create the particle being logged). Thus problems can be decomposed into stages, and were for this study. In the initial stage of each calculation, particles from the neutron source are transported to a bounding surface that encloses the detector, where they are logged. In the second stage, the logged particles are used as a virtual source and transported a short distance through air and into the bounded volume containing the candidate detector. With this staged approach, the most time-consuming first portion of the simulation need be performed only once, and then many detectors can be evaluated using relatively brief calculations that, in this study, gauge the reactions that determine detectability of the signature.

Figures $1 \mathrm{a}$ and $1 \mathrm{~b}$ below show different views of the model geometry used by COG. This geometry matches the generic physical arrangement at the LLNL cargo test bed. Thus results of the Monte Carlo simulations for this scenario could apply to actual experiments therein. The colors in the figures indicate different materials in the simulation (represented by densities and reaction cross section tables for the nuclides present). In general, the materials are mixtures of elements and may also be composites of mixtures. Where composite materials are assigned to the model regions, they are "smeared out" homogeneously over the region volume. For example, though the concrete contains steel reinforcing, the iron in the model is not concentrated into bars, but is distributed throughout in a fictitious average mixture.

The COG geometry model includes those elements of both the apparatus and the experiment hall, which influence the transport of particles from the source to the target and from the target to the detector. For example, it includes the floor,

\footnotetext{
${ }^{\dagger}$ An excellent example is the Nuclear Material Identification System (NMIS) developed at Oak Ridge National Laboratory.
} 
which reflects a detectable number of particles back into the experiment. The black box in Figure 1 encloses a volume large enough to accommodate all of the candidate detectors used in this study. This detector bounding volume is a $2 \times 2$ x 0.5 meter slab centered on a horizontal path to the target. All detector fluences and fluxes in this study are calculated per unit area of this six-sided surface.

We assume a slightly idealized neutron source for this problem that emits isotropically and is a "delta" $(\delta)$ function in both time and energy. In reality, the neutrons generated will have both a slight angular dependence and a narrow pulse width. However, since these widths are small compared to the time and energy scales over which the particles are to be resolved, we accept the $\delta$ functions as good approximations. We considered two source neutron energies, 2.45 and 14.1 $\mathrm{MeV}$, as D-D and D-T based neutron generators would produce. (The lower source energy offers the advantage of a greater energy range for accommodating the fission signature. However, we also wanted to assess the advantage in terms of interrogating flux on target that might accrue from using more penetrating source particles. Of course, the fission rate will also depend on how well thermalized are the arriving neutrons.) We represent the target as a spherical shell to make good use of the small amount of HEU mass that a typical experiment will be permitted to use. Since HEU is essentially a surface emitter, we can thereby approximate the signature from a more massive but solid sample of the material having the same radius.

We chose plywood to simulate cargo in the model because it contains the main constituents of a large fraction of known cargoes. Analyzing cargo manifests for a local port showed that the majority of cargoes sampled were rich in plant products, for example, vegetable produce and wood. The most prevalent molecule in both of these is cellulose, which is rich in hydrogen. Cellulose in the form of plywood makes a convenient substance for use in experiments, and has been used for this purpose in measurements taken at LLNL. Also, using laminated sheets of plywood as a cargo simulant improves reproducibility in experiments, and makes geometry models easy to construct-one can make accurate predictions using simpler 1-dimensional calculations.

To gauge the effect of simulating cargo with plywood, we repeated two calculations from the HEU detection study with combined layers of steel, aluminum, and plywood substituted for plywood alone. The atom density of the laminated steel-aluminum-plywood cargo matched the plywood-only case, and we chose the thickness of each substance to contribute one third of the total atom density. Though we held the atom density constant, the areal density increased from 29.5 to $78.5 \mathrm{~g} / \mathrm{cm}^{2}$

Lastly, we explored the effects on detection of partial streaming paths, due to voids in the cargo, by detailed modeling of 12-bottle cases of wine. We replaced the plywood simulant shown in green in Figure 1a with $7(\mathrm{w})$ x $8(\mathrm{~h}) \times 2(\mathrm{~d})$ cases of $4 \times 3$ bottles each, extending to approximately the same height and width dimension as the plywood. The cargo volume is $43 \%$ air. To quantify the effect of this spatial heterogeneity, we repeated the calculation using the same materials distributed in an average density approximation over the entire volume of the cases.

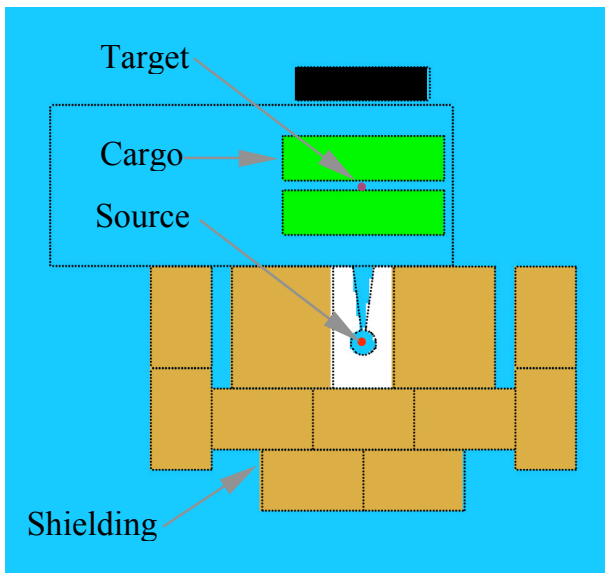

Figure 1a. Horizontal section at source elevation showing the main components of the problem, including he detector (black). The cargo thickness varies as part of this study, but its inner edge remains a constant distance from the target. Note that the cargo symmetrically surrounds the target.

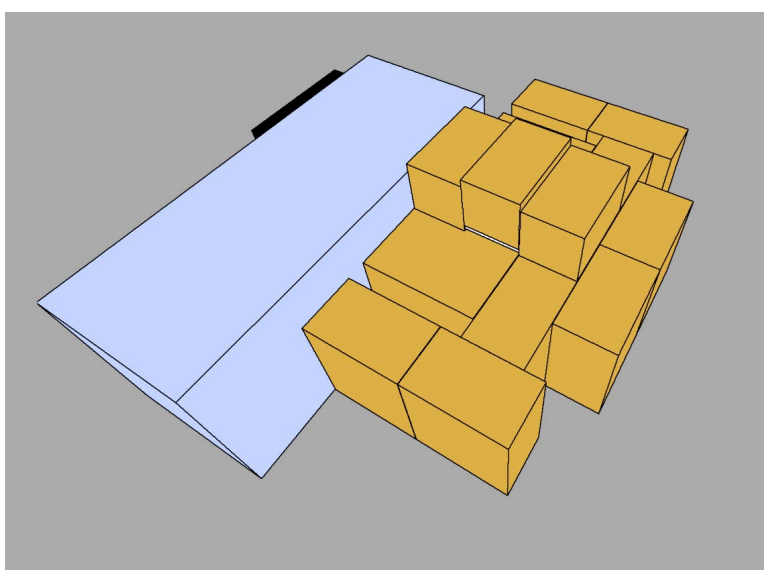

Figure 1b. Perspective rendering of the problem geometry. The center-to-center distance from source to detector is about $4 \mathrm{~m}$, approximately collinear with the source and target. The target is a hollow shell as one might arrange to increase the surface area in an experiment where the amount of HEU may be controlled. 


\section{RESULTS OF TRANSPORT CALCULATIONS}

Figure 2 below shows a false color representation of neutron fluence onto the wall of the cargo container adjacent to the collimator. Calculations confirm that as the neutrons enter the container volume, they are scattered, absorbed, and moderated in the volume of the simulated cargo. The fluence of neutrons actually reaching the target is shown in Figures $3 \mathrm{a}$ and $3 \mathrm{~b}$ as a function of energy, while treating as a parameter the areal density $(\rho \cdot \mathrm{L}$, where $\mathrm{L}$ is the cargo thickness separating the source from the target, and symmetrically the target from the detector). In both cases (and generally for this study) the fluence is normalized to a value per source neutron.

\section{Neutron Flux on Container Front Face}

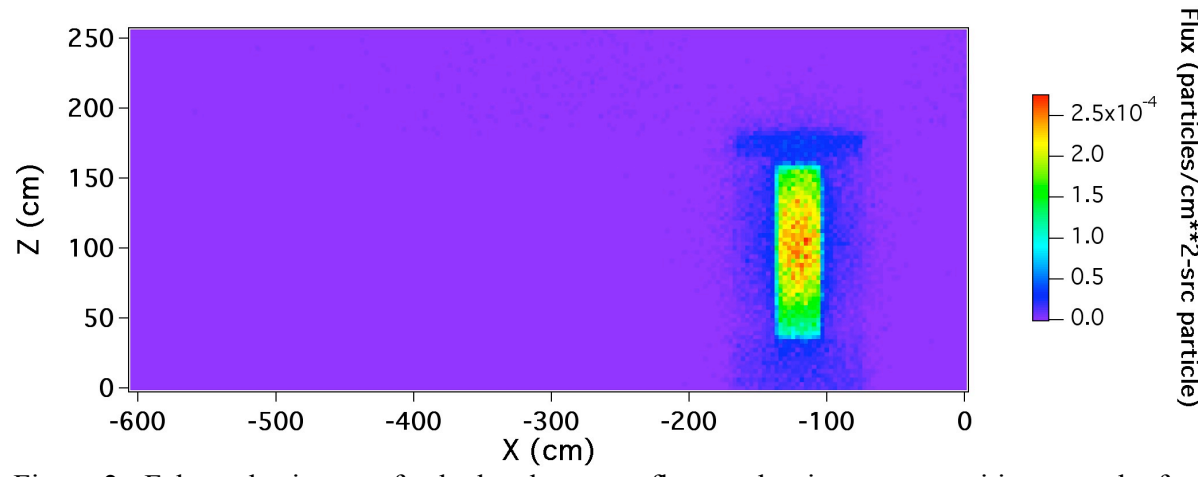

Figure 2. False color image of calculated neutron fluence density versus position over the front face of the cargo container, showing concentration of particles at the exit aperture of the collimator, i.e., good collimation. The faint blue horizontal bar over the top of the intense region corresponds to a deliberately left gap in the shielding material above the source that accommodates its power and cooling lines.

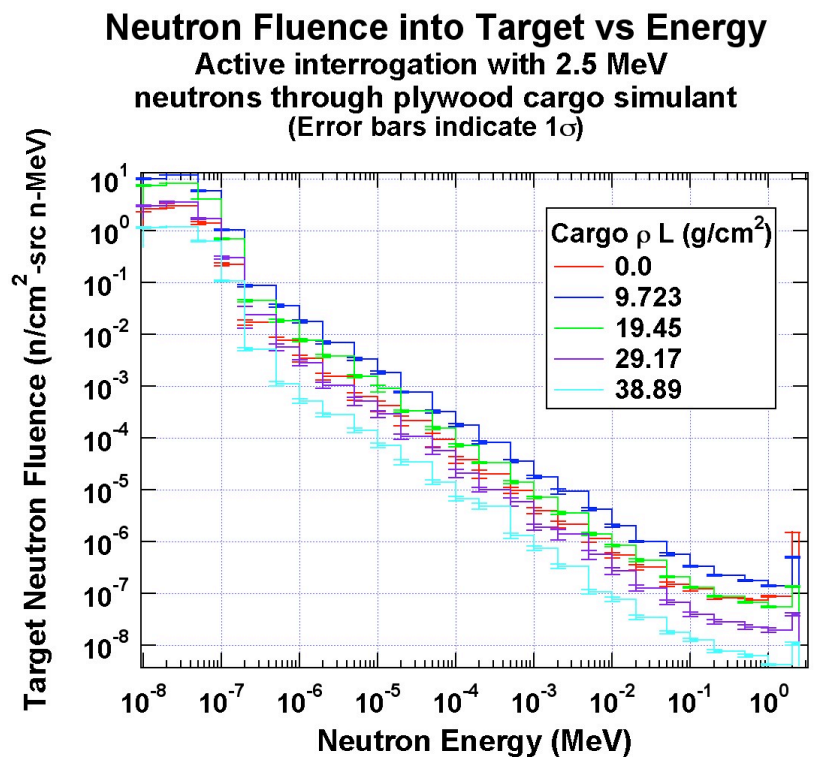

Figure 3a. Fluence spectrum across the surface of a perfectly absorbing version of the spherical target, for increasing values of $\propto \mathrm{L}$ Comparison to $\rho \mathrm{L}=0.0$ demonstrates the moderating effects of the cargo over and above the concrete shielding and floor, the polyethylene in the collimator, and the steel of the container.

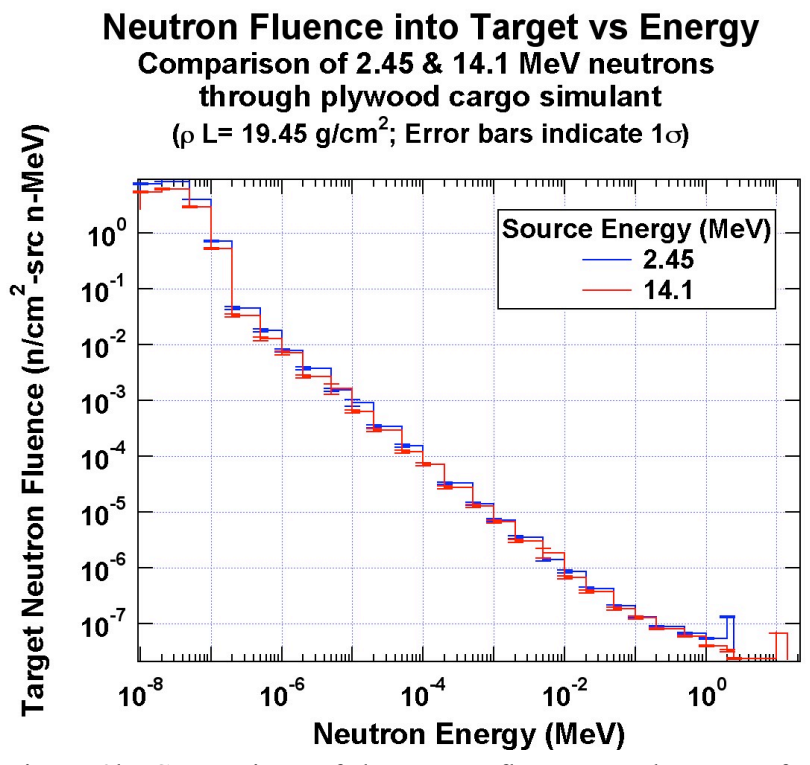

Figure $3 \mathrm{~b}$. Comparison of the source fluence on the target for the two different source energies used. Note that since the detector response in each energy bin was divided by the bin width, the uncollided fluence is greater at $14.1 \mathrm{MeV}$ than at $2.45 \mathrm{MeV}$.

The spectra in Figure 3a show that, for the cargo thicknesses we modeled, the spectrum of neutrons (source plus downscatter) is greatest in nearly all energy groups at $\rho \mathrm{L}=9.7 \mathrm{~g} / \mathrm{cm}^{2}$. We believe that this maximum reflects a combination of relatively low attenuation of the incident beam, and of neutron backscatter from cargo behind the target 
(that is, between the target and detector). In the case of the empty container, most of the neutron fluence arrives at the target unscattered, or minimally scattered. This case is thus underthermalized, and one should not expect as strong a signal due to fissions in the target as one would expect with a small or even moderate amount of cargo present. The reason for this is clear if we recall the energy dependence of the uranium fission cross section, which favors thermal and epithermal neutrons. At increased cargo thickness, the effect of attenuation clearly dominates the backscatter. The cited values of the cargo thickness (and $\rho \mathrm{L}$ ) refer to the equal amounts of cargo placed symmetrically between the source and the target and between the target and the detector, as shown in Figure 1.

Figure $3 \mathrm{~b}$ shows how the fluence on target is affected by increasing the interrogating neutron energy from $2.5 \mathrm{MeV}$ to 14.1 MeV. At the moderate cargo thickness corresponding to $\rho \mathrm{L}=19.45 \mathrm{~g} / \mathrm{cm}^{2}$, the relative spectral intensities differ by a small fractional amount, though the $14.1 \mathrm{MeV}$ source neutrons arrive at the target in greater numbers sooner. Nevertheless, as we expect, the energy- and time-integrated fluences into the target are close to one another: $9.6 \mathrm{x} 10^{-7}$ neutrons $\mathrm{cm}^{-2}$ source neutron ${ }^{-1}$ for $2.5 \mathrm{MeV}$ and $1.2 \times 10^{-6}$ neutrons $\mathrm{cm}^{-2}$ source neutron ${ }^{-1}$ for $14.1 \mathrm{MeV}$ source neutrons, but more of the higher-energy particles arrive at the target unscattered and thus unmoderated. The small relative differences in the spectrum of neutrons incident on the target led us to conclude that our findings for D-D source neutrons would apply more generally.

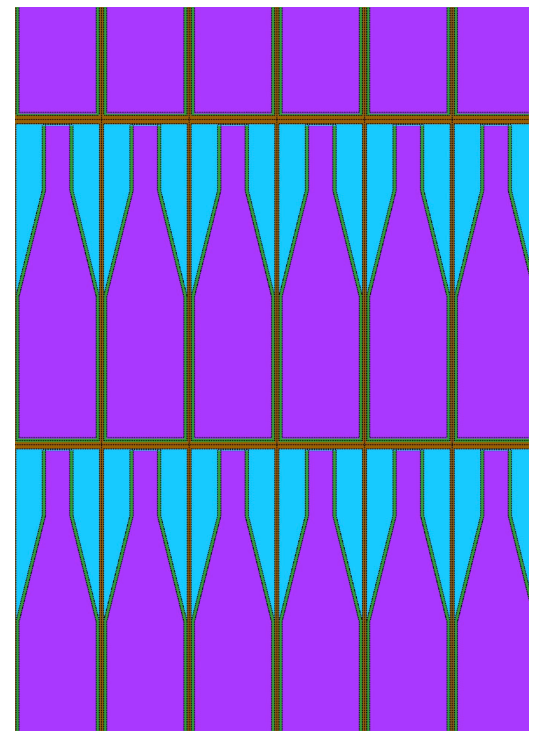

Figure 4a. Section through a vertical plane showing a portion of the stacked wine cartons modeled to evaluate the effects of streaming paths between the source and target, and target and detector. The model includes cardboard, air, glass, and water. The modeled cases subtend the same solid angle as the plywood cargo, with air paths accounting for $\sim 25 \%$ of the cross section.

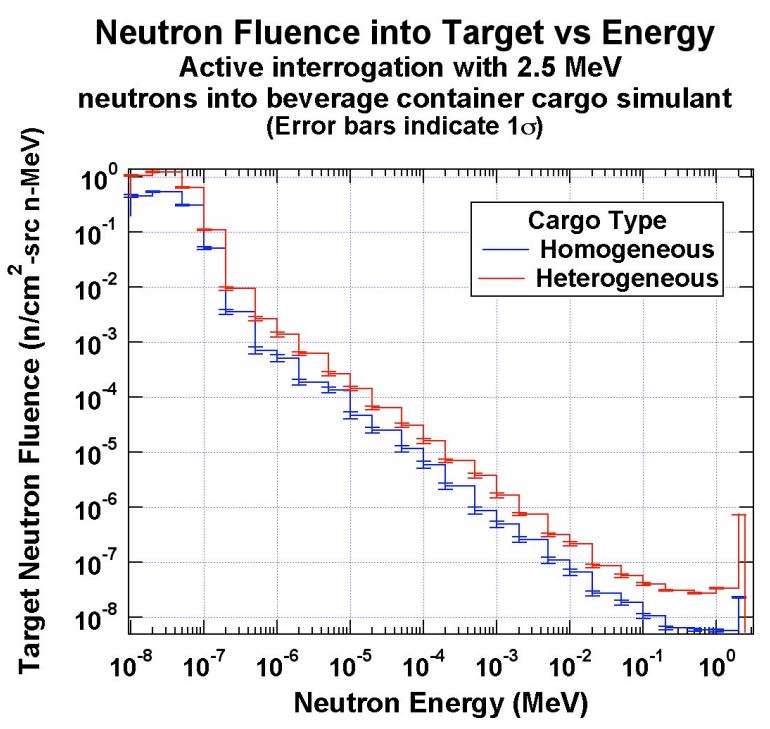

Figure $4 \mathrm{~b}$. Comparison of the fluence spectra across the surface of the perfectly absorbing target for the detailed wine case model illustrated in Figure $4 \mathrm{a}$, and a mean density model $\left(\rho=0.643 \mathrm{~g} / \mathrm{cm}^{3}\right)$ using the same materials averaged out over the same volume occupied by the wine cartons. Note the dominant weakly scattered component in the heterogeneous (streaming) case

Particles arriving at the detector's bounding surface are logged into a census file for use in the detector model. The logged properties include the age and the energy of the particles. The flux plots in Figures 5a and 5b compare these properties for particles logged into the census files as they cross the surface of the detector bounding volume. The calculation plotted in Figure 5a used an HEU target, while the one plotted in 5b used a null target. The two calculations used identical input, including the HEU target, except that in the null case all fission reactions were suppressed. The comparison shows several differences, particularly a cluster of neutrons at and below fission energies at times on the order of $1 \mathrm{~ms}$ in the HEU case. Similarly, a more moderated cluster occurs at times characteristics of delayed fission in the HEU case, clustering around the thermal peak at time $\mathrm{t}>50 \mathrm{~ms}$.

The regions enclosed by dashed lines in Figure 5a identify two potential signatures. Note the large disparity in intensity of the thermal flux due to all processes except fission and the identified signatures. This underscores the need for crisp energy discrimination in a detector meant to exploit the higher energy, medium age range $(0.1 \leq t \leq 10 \mathrm{~ms}$, triangular 
region) signature, and/or high contrast (low leakage between pulses) in the neutron source for systems meant to exploit the late time $(t>100 \mathrm{~ms}$, rectangular region) signature.

\section{Neutron Flux as Function of Energy and Time} (HEU Target; $\rho \mathrm{R}=\mathbf{1 9 . 4 5} \mathrm{g} / \mathrm{cm}^{2}$ )
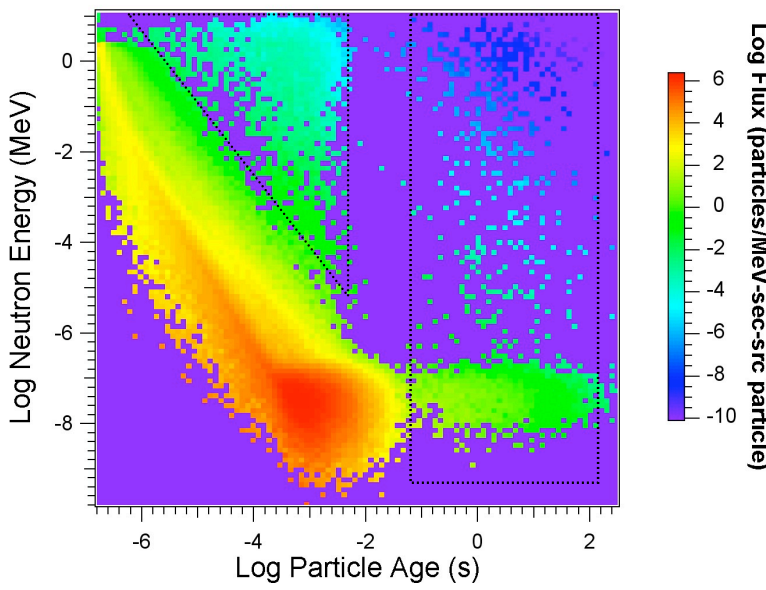

Neutron Flux as Function of Energy and Time (Null Target; $\rho R=19.45 \mathrm{~g} / \mathrm{cm}^{2}$ )

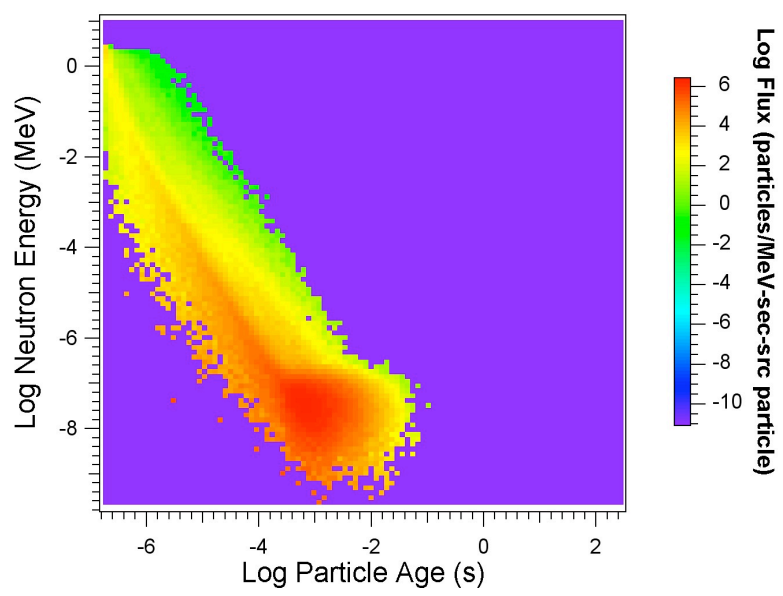

Figures 5a \& 5b. Neutron flux plots rendered over the energy-time domain of particles crossing the $2 \mathrm{~m} \times 2 \mathrm{~m} \times 0.5 \mathrm{~m}$ detector bounding surface, for calculations with HEU (left) and null (right) targets. (The null target is simply HEU in which fission is turned off.) Note the contributions from the fission reactions. The dashed triangle and rectangle enclose regions of the domain at highenergy and late-time containing the HEU signatures of interest. The false color values are derived by dividing this domain into discrete intervals (i.e., rectangular tiles) and scaling the particle fluence into each interval by its extent in energy and time. (Note that in some cases, the flux value within an energy-time interval is insufficient to guarantee statistical significance.) In this way we can compare the contribution per source neutron across the domain and better gauge the strengths of the candidate fission signatures with respect to the source pulse.

In the corresponding analysis of gamma rays shown in Figure $6 \mathrm{a}$ and $6 \mathrm{~b}$, the emission of gamma rays beyond $50 \mathrm{~ms}$ in the HEU case indicates radiation that originated with delayed neutrons, and was created as a result of capture or promptly emitted from subsequent fissions.
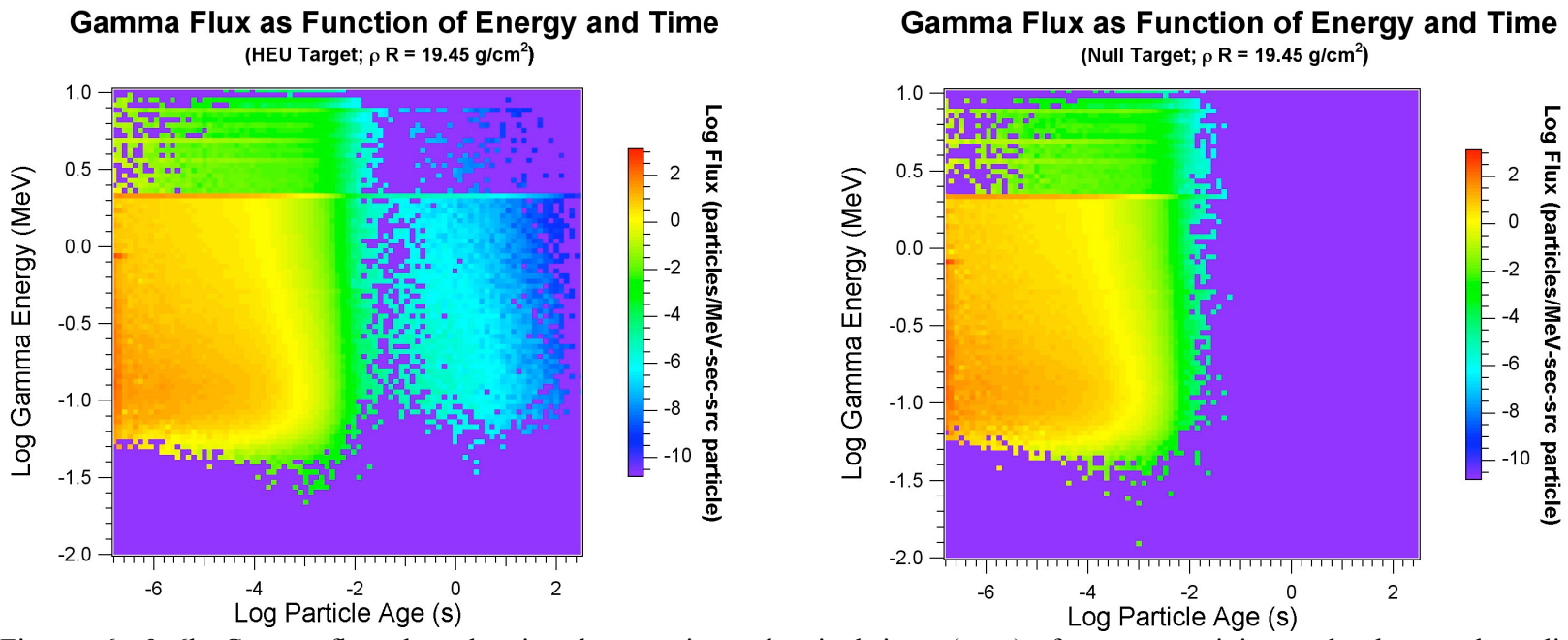

Figures $6 \mathrm{a} \& 6 \mathrm{~b}$. Gamma flux plots showing the energies and arrival times (ages) of gammas arriving at the detector bounding surface, for calculations with HEU (left) and null (right) targets. Note in 6a the persistence in time of the hydrogen thermal neutron capture line at $\mathrm{E}=2.22 \mathrm{MeV}(\log \mathrm{E}=0.346)$. This might serve as a usable indicator of the presence of delayed neutrons due to HEU fission coming from the surveyed volume. Other prominent lines predicted by both simulations are from oxygen $(0.871$ $\mathrm{MeV}, 2184.47 \mathrm{MeV}$ ), carbon (3.683 MeV, 4.945 MeV), iron (7.631 MeV, 7.645 MeV, 9.234 MeV), and silicon (3.539 MeV and $4.934 \mathrm{MeV}$ ). 
Neutron Flux as Function of Energy and Time (HEU Target; Wine Case Cargo)

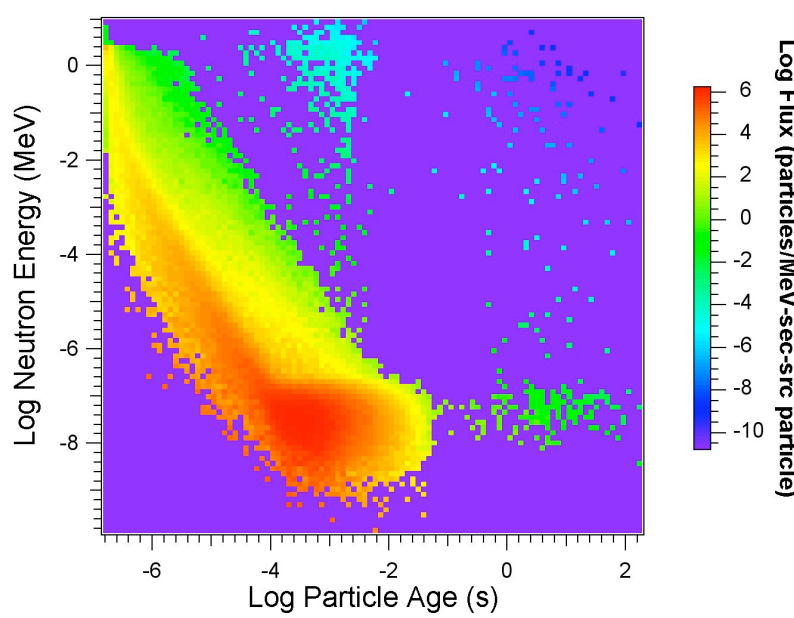

Neutron Flux as Function of Energy and Time (HEU Target; Average Density Model)

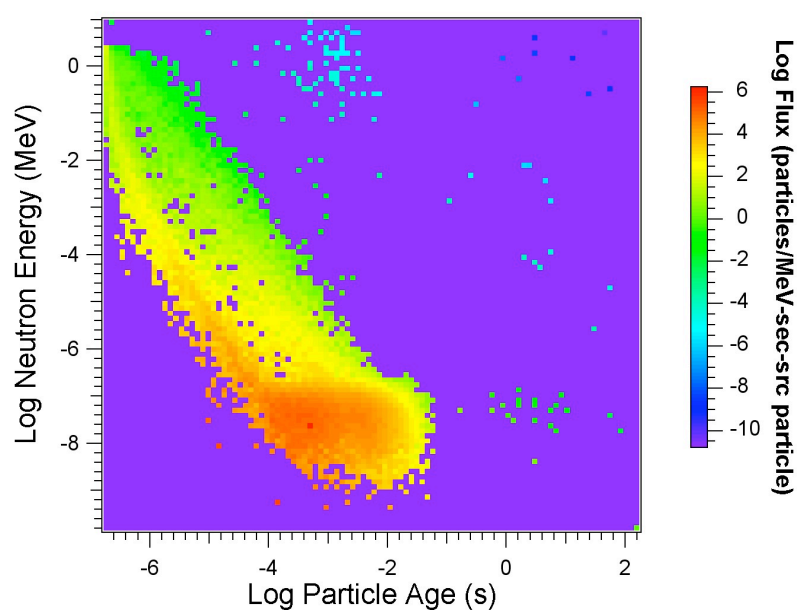

Figure $7 \mathrm{a} \& 7 \mathrm{~b}$. Neutrons crossing the detector bounding surface for cargo consisting of simulated cases of wine (a), and an equivalent average density representation (b) intended to show the effect of streaming paths on the intensities of the candidate signatures. We postulate that attenuation due to scattering reduces the intensity in both cases, while lack of moderation also reduces the streaming path signature.

Figures $7 \mathrm{a}$ and $7 \mathrm{~b}$ show the effects of spatial heterogeneity (voids) on both candidate fission signatures. In $7 \mathrm{a}$, some neutrons are able travel through air, and a large fraction arrive at the target either uncollided, or at energies above 1 $\mathrm{MeV}$, where the neutron scattering cross section of hydrogen declines sharply. Comparing these results to the plywood cargo calculation (Figure 5a), we observe that both fission neutron signatures are weakened even at a comparable average $\rho \mathrm{L}\left(32.8 \mathrm{~g} / \mathrm{cm}^{2}\right)$. We attribute the difference to the higher specific hydrogen content of the wine case model.

\section{DESCRIPTION OF THE DETECTOR MODEL}

In the second stage of detector analysis, the particles previously logged into the census file are regenerated and tracked as new particle histories. While the volume within the detector bounding surface in the first stage of the model is defined to be a perfect absorber, in the second stage it contains the particular detectors being analyzed. Outside the bounding surface remains the full model geometry (collimator, cargo container, cargo, floor, walls, etc.). Two detectors have been modeled inside the bounding surface. The first is a calcium fluoride crystal, chosen primarily because, in theory, it can be configured to have a neutron detection threshold above the D-D neutron interrogating energy of 2.45 $\mathrm{MeV}$ but remain sensitive to roughly $30 \%$ of the typical fission neutron spectrum. ${ }^{*}$ The second detector type is a large organic scintillator, representing a hydrogenous proton-recoil detector.

A key concern in the use of both the crystal and liquid scintillators is the ability to discriminate between neutroninduced charged particles (protons and alpha particles for the $\mathrm{CaF}_{2}$ and recoil protons for the liquid) and gamma-ray induced electrons (primarily from Compton scattering). The gamma-ray flux is expected to be intense during active neutron interrogation due to capture reactions in materials such as hydrogen, nitrogen, and iron, and due also to inelastic scatter reactions in light nuclei. For this limited analysis, we will not simulate the process of gamma-ray discrimination in the $\mathrm{CaF}_{2}$ crystal or the liquid scintillator, but rather investigate the necessary discrimination capability required for fissile material detection.

Detector specifications for both scintillators are provided below in Table I. Note that the thickness of the $\mathrm{CaF}_{2}$ crystal has been limited to $2 \mathrm{~cm}$ to provide a conservatively low estimate of feasible crystal size.

\footnotetext{
${ }^{*}$ Based on a Watt fission spectrum with parameters $\mathrm{a}=0.988$ and $\mathrm{b}=2.249$ for thermal neutron induced fission in ${ }^{235} \mathrm{U}$.
} 
Table I. Specifications for calcium fluoride- and liquid organic-scintillator models.

Calcium Fluoride Scintillator

\begin{tabular}{|lll|lll|}
\hline Composition: & & Composition: & $\mathrm{C}\left(\mathrm{g} \mathrm{cm}^{-3}\right)$ & 0.915 \\
& ${ }^{19} \mathrm{Ca}\left(\mathrm{g} \mathrm{cm}^{-3}\right)$ & 1.7 & & $\mathrm{H}$ & 0.085 \\
& Total density & 1.5 & & Total density & 1.03 \\
\hline Geometry: & Height $(\mathrm{cm})$ & 200 & Geometry: & Height $(\mathrm{cm})$ & 200 \\
& Width & 200 & & Width & 200 \\
& Depth & 2 & & Depth & 15 \\
\hline Reactions: & ${ }^{\text {nat }} \mathrm{Ca}(\mathrm{n}, \mathrm{p})$ & $2.5 \mathrm{MeV}+(\mathrm{Q}=-0.53 \mathrm{MeV})$ & Reactions: & Elastic scatter on $\mathrm{H}$ & $\mathrm{E}_{\mathrm{n}}>1 \mathrm{MeV}$ \\
& ${ }^{\text {nat }} \mathrm{Ca}(\mathrm{n}, \alpha)$ & $2.5 \mathrm{MeV}+(\mathrm{Q}=+1.75 \mathrm{MeV})$ & & Compton scatter & $\mathrm{E}_{\mathrm{g}}>0.1 \mathrm{MeV}$ \\
& ${ }^{19} \mathrm{~F}(\mathrm{n}, \mathrm{a})$ & $2.5 \mathrm{MeV}+(\mathrm{Q}=-1.52 \mathrm{MeV})$ & & & \\
\hline
\end{tabular}

\section{DISCUSSION OF DETECTOR RESPONSE}

The detection response of the calcium fluoride crystal detector is taken to be the sum only of the induced $\mathrm{Ca}(\mathrm{n}, \alpha)$ and $\mathrm{Ca}(\mathrm{n}, \mathrm{p})$ reactions. The cross sections for these reactions, as well as the $(\mathrm{n}, \alpha)$ reaction in ${ }^{19} \mathrm{~F}$, are provided in Figure 8 .

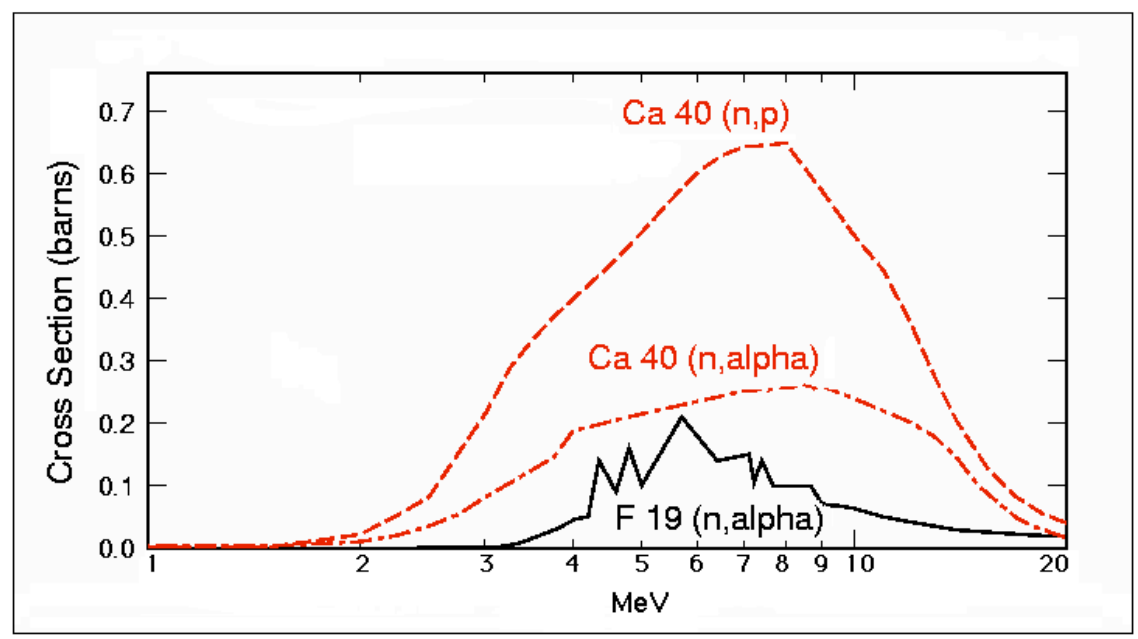

Figure 8. Reaction cross sections. All data come from ENDFB6

Because the cross section, $\mathrm{Q}$ value, and light conversion for the $(n, \alpha)$ reactions in fluorine are relatively poor compared to the reactions in calcium, all fluorine reactions will be neglected for this study. Other critical assumptions apply to the crystal as modeled. Notably, gamma-ray induced reactions have been omitted for the crystal, light conversion and collection have not been simulated, and we have not accounted for associated inefficiencies.

The detection response of the proton-recoil liquid scintillator is approximated by the number of proton-recoil events in the organic detector. Given our inability to distinguish between multiple elastic scatterings from a single neutron, the simulated results represent a conservative upper bound on the actual reaction rate. Only recoil events due to incident neutron energies above $1 \mathrm{MeV}$ are considered. As with the $\mathrm{CaF}_{2}$ crystal, light conversion and collection have not been considered.

We have also estimated the number of Compton electron producing reactions in the scintillators in order to gauge the level of gamma-ray discrimination required for the threshold neutron detection. Results are provided for the organic scintillator but are representative of the $\mathrm{CaF}_{2}$ crystal as well. Only the Compton scatterings of gamma rays above 100 $\mathrm{keV}$ are considered, reflecting the higher light conversion efficiency of the Compton electrons as compared to heavier ions. $^{5}$

Simulated reaction rates in the detectors must be considered in the context of the cosmogenic neutron background flux. The cosmic ray neutron flux at sea level, due to the interaction of high-energy cosmic rays with the environment, is generally taken as $1 \times 10^{-2} \mathrm{n} \mathrm{cm}^{-2} \mathrm{~s}^{-1}$. Of this, $4 \times 10^{-3} \mathrm{n} \mathrm{cm}^{-2} \mathrm{~s}^{-1}$ is assumed thermal $\left(0<\mathrm{E}_{\mathrm{n}}<0.4 \mathrm{eV}\right), 4 \times 10^{-3} \mathrm{n} \mathrm{cm}^{-2} \mathrm{~s}^{-1}$ is assumed epithermal to fission-energy $\left(0.4 \mathrm{eV}<\mathrm{E}_{\mathrm{n}}<1 \mathrm{MeV}\right)$, and $2 \times 10^{-3} \mathrm{n} \mathrm{cm}^{-2} \mathrm{~s}^{-1}$ is assumed to be fast $\left(\mathrm{E}_{\mathrm{n}}>1 \mathrm{MeV}\right)$. 
For the purposes of this analysis, all detection events from cosmic background are taken to be from the fast energy group.

In order to estimate the count rate due to cosmic ray neutrons, one must first estimate the detector efficiency in the fast energy range. The Monte Carlo results indicate combined $(n, p)$ and $(n, \alpha)$ reaction rates in calcium of $1.5 \times 10^{-10}$ sourceparticle $\mathrm{cm}^{-3}$ for an incident neutron flux of $1.5 \times 10^{-7} \mathrm{n}$ source-particle ${ }^{-1} \mathrm{~cm}^{-2}$ in the energy range $0.5 \mathrm{MeV}$ to $15 \mathrm{MeV}$. Because the cosmic ray neutron spectrum drops sharply with increasing energy, the limited energy range is a reasonably conservative estimate of the detectable fast portion of the spectrum. The resulting macroscopic cross section is 0.001 $\mathrm{cm}^{-1}$. If we take $1 \times 10^{-3} \mathrm{n} \mathrm{cm}^{-2} \mathrm{sec}^{-1}$ as an estimate of the cosmic ray neutron flux between 1 to $10 \mathrm{MeV}$, the detected cosmic ray reaction rate is roughly $1 \times 10^{-6} \mathrm{~cm}^{-3} \mathrm{~s}^{-1}$. As the results will show, this is many orders of magnitude less than the peak rates of detection for induced prompt fission neutrons for the given D-D neutron interrogations.

\section{RESULTS OF DETECTOR CALCULATIONS}

Below we present results for both the HEU and null targets. The HEU target may emit induced prompt fission neutrons and gamma rays, as well as delayed fission neutrons. In the null case all fission reactions in the HEU are suppressed. Results are provided for the two types of cargos described in Section 2: plywood of moderate and high areal density and a low-atomic mass material representative of palletized wine bottles (in both a lattice or "heterogeneous" geometry and a uniform density "homogenous" geometry).

To assist with interpreting the results, a few clarifications may be helpful. First, note that model results are presented in histogram format, showing the number of selected reactions in the detector for a given time period. The results are not reaction rates and therefore need not be multiplied by the time interval to obtain fluence. Second, the reported uncertainties are derived from Monte Carlo precision only and are not predictions of statistical variations from experiments. Finally, reaction rates are provided in terms of the number of reactions per single interrogation pulse, and per unit detector volume if so specified.

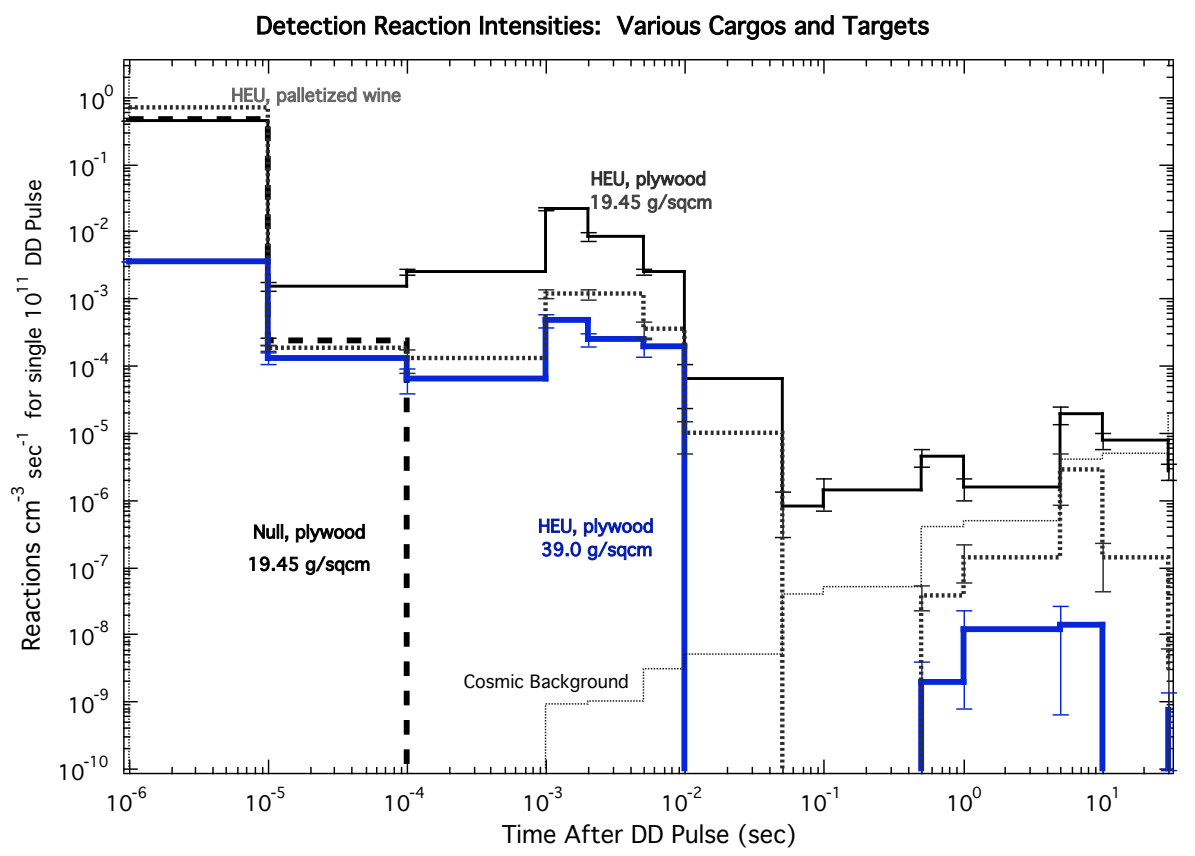

Figure 9: Histogram of Reactions as a function of time after interrogation pulse, for DD neutron interrogation. Curves represent the $\mathrm{CaF}_{2}$ responses for the base null case $\left(19.45 \mathrm{~g} \mathrm{~cm}^{-2}\right)$, and fissionable HEU target cases for base $\left(19.45 \mathrm{~g} \mathrm{~cm}^{-2}\right)$, thick loading $\left(39 \mathrm{~g} \mathrm{~cm}^{-2}\right)$, heterogeneous low-z (palletized wine bottles).

Figure 9 shows the results for various calculations as well as the estimated cosmogenic background reaction intensity. The null case displayed is for the $19.45 \mathrm{~g} \mathrm{~cm}^{-2}$ plywood loading, but is representative of all null cases, in that no reactions are predicted beyond $100 \mu$ s from the neutron pulse. In all HEU (fissioning) cases, the peak reaction intensity (ignoring the initial source pulse) occurs at 1 to $5 \mathrm{~ms}$, and falls abruptly after $50 \mathrm{~ms}$. Reactions occurring after $0.1 \mathrm{~s}$ 
show predicted delayed neutron activity. Note that the variances in the Monte Carlo results in this latter region are large, and reaction rates are below the estimated background rates. This suggests that robust detection of the delayed signature would require long counting times to increase detection confidence at reasonable false alarm rates.

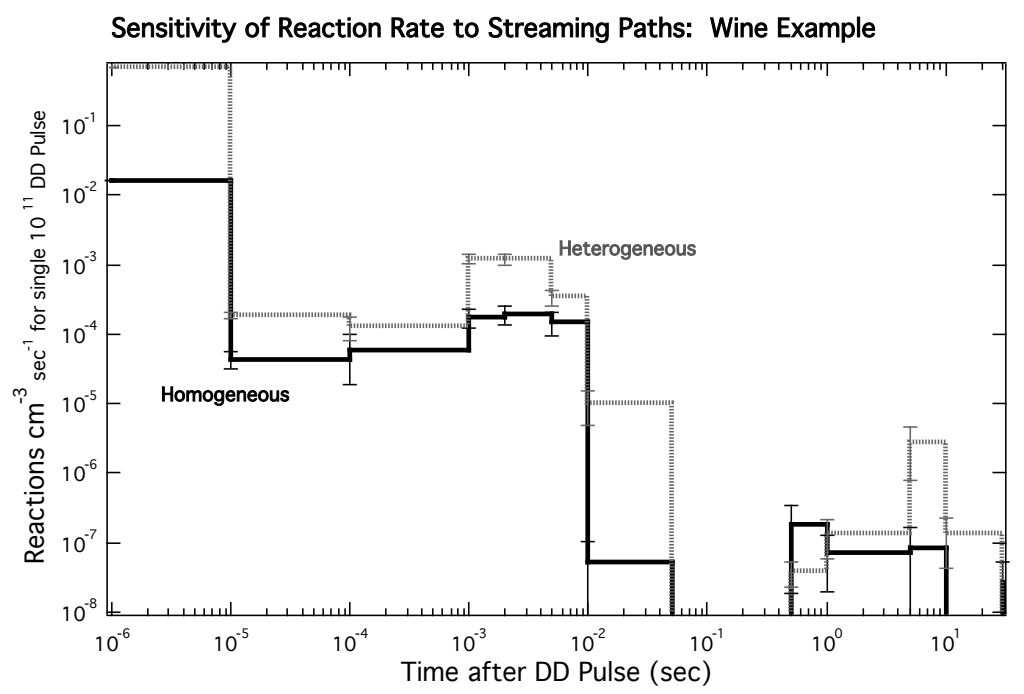

Figure 10: Histogram of reactions as a function of time after interrogation pulse, for D-D neutron interrogation. Curves represent the $\mathrm{CaF}_{2}$ responses for the heterogeneous low- $\mathrm{Z}$ case (Figure 7a) and the homogenous low- $Z$ case (Figure $7 \mathrm{~b}$ ). The results show the importance of considering the effect of streaming paths in assessing the feasibility of the active interrogation method.

Figure 10 illustrates the effect of cargo geometry on the predicted reaction rates. Both histograms report data of fission cases with simulated wine cargo. In the homogeneous case, all cargo materials have been combined into a representative cargo volume of uniform density. In the heterogeneous case, individual bottles, their contents, and shipping boxes are modeled discretely. This allows for paths of "neutron streaming" into and out of the container. The result, for this particular case, is a predicted increase in reaction intensities for the heterogeneous model, likely due to decreased attenuation of fission neutrons along streaming paths.

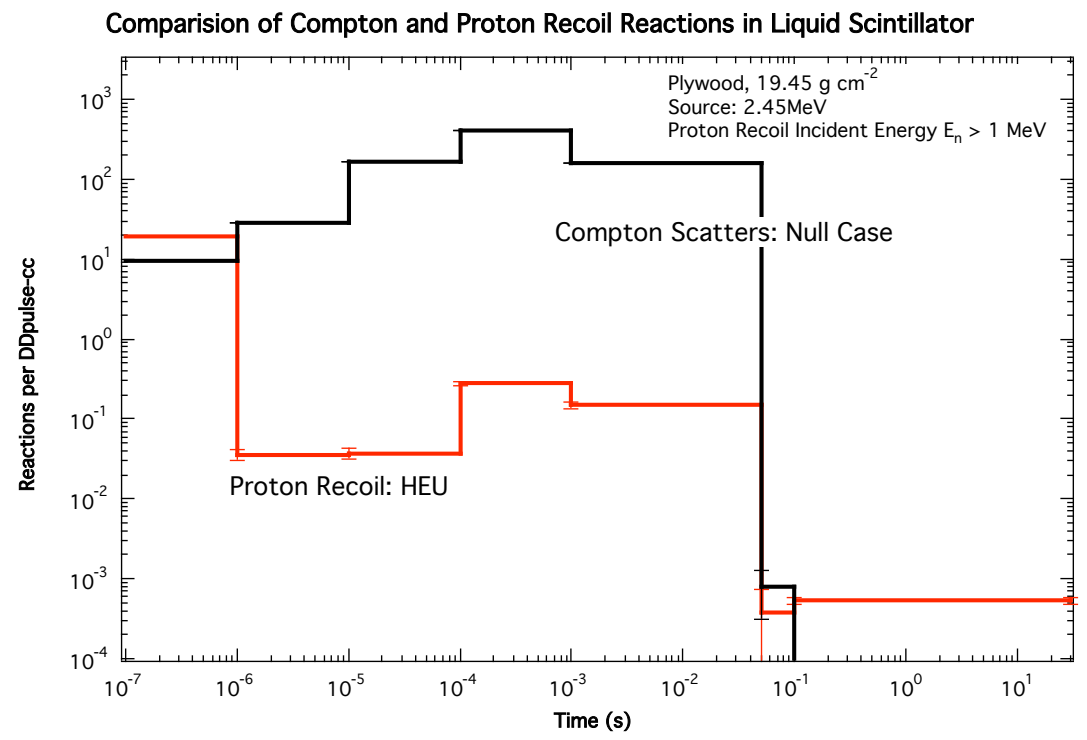

Figure 11. Histogram of calculated number of neutrons in liquid scintillator detector with energy $E_{n}>1 \mathrm{MeV}$ and number of Compton scatterings due to gamma rays with energy $E_{g}>$ $0.1 \mathrm{MeV}$, as a function of time after pulse, for a single D-D neutron pulse of $10^{11}$ neutrons. 
Finally, Figure 11 illustrates the importance of gamma-ray discrimination in the feasibility of either detection method considered. Results are shown for the organic scintillator, but are clearly also relevant for the $\mathrm{CaF}_{2}$. The histograms show that the predicted intensity of Null case Compton electrons $\left(\mathrm{E}_{\text {gamma }}>100 \mathrm{keV}\right)$ is 2 to 3 orders of magnitude greater than the proton recoil intensity $\left(\mathrm{E}_{\text {neutron }}>1 \mathrm{MeV}\right)$ in the HEU case. This suggests that, for the given conditions, a signature based on prompt fast neutrons will require particle discrimination with roughly $99.9 \%$ effectiveness. We believe that this may be the key challenge in making this active interrogation approach viable, regardless of the particular detector.

\section{CONCLUSIONS AND SUGGESTIONS FOR FURTHER WORK}

\section{Conclusions:}

1. Neutrons from induced prompt fission of HEU may be a robust signature, when combined with time gating, threshold detection and stringent gamma-ray discrimination. In this study, the simulated signatures of fissile material for the modeled configurations were remarkably robust after the 0.1 to $50 \mathrm{~ms}$ time interval.

2. Delayed neutrons and associated gammas may also offer a viable signature, requiring the use of time-gating methods, in which the detector signal is ignored for a characteristic time interval after the source pulse. This signature may require a very large detector.

3. Gamma ray discrimination will be a key hurdle in developing a feasible active interrogation threshold detection system. The gamma-ray flux induced by the interrogating neutron beam is far more intense-by 2 to 3 orders of magnitude-than the threshold prompt neutron flux. Highly robust and effective discrimination methods will be required to discern neutron reactions from gamma rays from capture and inelastic scatter in the cargo and environment.

4. With its infinite range of possible contents, a sealed cargo container is a "black box," requiring careful analysis of all available information to screen it. Detectors can be designed and algorithms can be made to filter distracting signals, but only if such signals can be isolated. A useful instrument must tolerate a broad range of conditions affecting the presence and quality of HEU signatures. Possibly, no single indication alone will suffice to reliably distinguish suspicious from legitimate cargo, without a high rate of false positives. One might need to combine several signatures to increase the probability of correctly classifying the interrogated object.

\section{Future Work:}

1. In view of our simulation results, a prudent next phase would feature experiments with the outlined interrogation system. These experiments would help validate these modeling results and disclose any overlooked physics or incorrect assumptions. Sources of possible disagreement with the model include the cross section tables and simplifying assumptions about the geometry and materials involved.

2. In this analysis we predicted the number of detectable reactions in notional detectors for single interrogation pulses. To model the number of counts in an actual glass or liquid scintillator, light pulse conversion, light collection, and charge conversion must be included in the analysis.

3. This study includes a preliminary critique of the detection method. Refining and expanding the scope of this critique will require simulations with finer resolution in time and energy, in turn requiring more particle histories, using variance reduction techniques for more quickly converging results.

4. Future modeling should test the assumption of "perfect" gamma-ray discrimination in the lithium fiber detector and extend the present study to consider problems of pulse pile-up and dead-time in the detectors and associated electronics.

5. New models should explore ways to limit the gamma-ray flux into the liquid scintillator, since gammas give rise to Compton electrons, which reduce the detector's ability to discriminate. The requirements for pulse shape discrimination in the liquid scintillator should be established beforehand.

6. Future research should explore ways of exploiting the persistence of the $2.22 \mathrm{MeV}$ gamma-ray line (and any other gamma rays indicative of prompt or delayed fission) in the time period 0.1 to $30 \mathrm{~s}$ after the source pulse. 


\section{ACKNOWLEDGMENTS}

This work was completed by Lawrence Livermore National Laboratory for the U.S. Department of Energy under contract W-7405-ENG-48.

\section{REFERENCES}

1. Slaughter, D., et al., Detection of Special Nuclear Material in Cargo Containers using Neutron Interrogation, Lawrence Livermore National Laboratory, UCRL-ID-155315 (August 2003).

2. Buck, R., Lent, E., Wilcox, T., COG-A Multiparticle Monte Carlo Transport Code, User's Manual, $5^{\text {th }}$ ed., LLNL (2002).

3. McCracken, D.D., "The Monte Carlo Method," Scientific American, 192 (1955), p. 90.

4. Bicron Corporation, Calcium Fluoride (Europium) Scintillators specification sheet \#3105(06-02).

5. Knoll, G.F. Radiation Detection and Measurement, 3rd ed. (2000), pp. 225-27. 